\title{
Preparation of hemispherical polystyrene particles utilizing the solvent evaporation method in aqueous dispersed systems
}

\author{
Takuya Tanaka ${ }^{1}$, Tomoe Yamagami ${ }^{1}$, Tatsuhiro Nogami ${ }^{1}$, Hideto Minami ${ }^{1}$ and Masayoshi Okubo ${ }^{1,2}$ \\ Various nonspherical polystyrene (PS) particles were prepared by slow evaporation of toluene (used common good solvent) from \\ homogeneous PS/hexadecane (HD)/toluene droplets dispersed in surfactant aqueous solutions at room temperature, followed by \\ the rapid removal of HD from PS/HD particles with various phase-separated morphologies. The morphology of PS/HD particles \\ was controlled by tuning the interfacial tension with various types of surfactants. Hemispherical PS particles with flat surfaces \\ were obtained from phase-separated PS/HD/toluene droplets having a Janus structure, when polyoxyethylene nonylphenyl ether \\ with an average ethylene oxide chain length of 30.8 was used as the surfactant.
}

Polymer Journal (2012) 44, 1112-1116; doi:10.1038/pj.2012.71; published online 23 May 2012

Keywords: nonspherical particles; hemisphere; solvent evaporation method; phase separation; interfacial tension; polystyrene

\section{INTRODUCTION}

Nonspherical polymer particles have attracted great attention because of their potential as materials with unique crystal structures, ${ }^{1-3}$ light scattering properties ${ }^{4}$ and external field-responsive properties (e.g., shear field ${ }^{5}$ and electric field ${ }^{6}$ ). In general, polymer particles synthesized by heterogeneous polymerizations under thermodynamic control have a spherical shape because of interfacial free energy minimization. However, nonspherical polymer particles have been synthesized under kinetic control utilizing various seeded polymerization methods, ${ }^{7-24}$ microfluidic techniques, ${ }^{25-28}$ deformation of spherical polymer particles by external force, ${ }^{29-31}$ the stepwise heterocoagulation method $^{32,33}$ and the self-organized precipitation method. ${ }^{34}$

In a previous work, we proposed a novel approach for the preparation of micrometer-sized, monodisperse, nonspherical (i.e., dimpled and hemispherical) polystyrene (PS) particles by successive heating and cooling of spherical PS particles dispersed in a methanol/ water medium in the presence of droplets of decane..$^{35}$ Decane was absorbed by the particles during heating, and the particles subsequently phase-separated into PS and decane phases during cooling. Eventually, nonspherical particles reflecting the morphology of phase-separated PS/decane particles were formed by rapid removal of the decane phase. The final particle shape could be controlled simply with the amount of absorbed decane.

We have also recently demonstrated morphology control of PS/poly(methyl methacrylate) composite particles prepared by the slow release of toluene as a common good solvent from homogeneous PS/poly(methyl methacrylate)/toluene droplets dispersed in a surfactant aqueous solution at room temperature (the solvent evaporation method) ${ }^{36-38}$ It was revealed that the particle morphology could be controlled by tuning the interfacial tensions by varying the types of surfactant.

In this article, we attempt to prepare various nonspherical (especially hemispherical) PS particles based on the above two experimental results. This will be achieved by morphology control of PS/hexadecane (HD) particles obtained by the solvent evaporation method (using toluene as the common good solvent) using various types of surfactants, followed by a rapid removal of the HD phase. $\mathrm{HD}$ is employed rather than decane because it will not evaporate during the toluene evaporation process.

\section{EXPERIMENTAL PROCEDURE}

\section{Materials}

Styrene was distilled under reduced pressure in a nitrogen atmosphere. Reagentgrade 1-pyrenylmethyl methacrylate (PM; Funakoshi, Tokyo, Japan) was used as a PS fluorescent moiety without further purification. Reagent-grade 2,2'-azobisisobutyronitrile (AIBN; Wako Pure Chemical Industries, Ltd., Osaka, Japan) was purified by recrystallization from methanol. Toluene and SDS were used as received from Nacalai Tesque, Inc (Kyoto, Japan). Poly(vinyl alcohol) (PVA; Gohsenol GH-17: degree of polymerization, 1700; degree of hydrolysis, $88 \%$ ) was supplied by Nippon Synthetic Chemical (Osaka, Japan). Commercial-grade polyoxyethylene nonylphenyl ether non-ionic surfactants with average ethylene oxide chain lengths of 15.4, 30.8 and 50.6 units (Emulgen 930, Emulgen 931 and Emulgen 950, respectively) were supplied by Kao Co., Tokyo, Japan. Water was purified using an Elix UV system (Nihon Millipore K.K., Tokyo, Japan).

Preparation of nonspherical particles

PS and S-PM copolymer (P(S-PM)) were synthesized by solution polymerization using 2,2'-azobisisobutyronitrile as an initiator. PS: $M_{\mathrm{w}} 8.8 \times 10^{4}$ $M_{\mathrm{w}} / M_{\mathrm{n}}, 2.1 . \mathrm{P}(\mathrm{S}-\mathrm{PM})(\mathrm{PM}, 0.5 \mathrm{~mol} \%): M_{\mathrm{w}}, 7.1 \times 10^{4} ; M_{\mathrm{w}} / M_{\mathrm{n}}, 1.7 . \mathrm{PS} / \mathrm{HD}$

${ }^{1}$ Graduate School of Engineering, Kobe University, Rokko, Nada, Kobe, Japan and ${ }^{2}$ Smart Spheres Workshop Co., Ltd., 2-1-214-122, Koyo-Naka, Higashi-Nada, Kobe, Japan Correspondence: Professor M Okubo, Graduate School of Engineering, Kobe University, Rokko, Nada, Kobe 657-8501, Japan.

E-mail: okubo@kobe-u.ac.jp

Received 23 January 2012; revised 6 March 2012; accepted 8 March 2012; published online 23 May 2012 
and $\mathrm{P}(\mathrm{S}-\mathrm{PM}) / \mathrm{HD}$ particles were prepared as follows: a homogeneous toluene solution of PS (or P(S-PM)) (0.643 g) and HD (PS (or P(S-PM))/HD/ toluene $=1 / 1 / 29, \mathrm{v} / \mathrm{v} / \mathrm{v}$ ) was mixed with a $0.33 \mathrm{wt} \%$ (relative to water) surfactant aqueous solution $(15 \mathrm{~g})$. The mixture was stirred vigorously using a homogenizer (NIHONSEIKI KAISHA Ltd., ABM-2 Tokyo, Japan) at 4000 r.p.m. for $2 \mathrm{~min}$ in a glass vial and toluene was then slowly released by evaporation during gentle stirring at room temperature for $24 \mathrm{~h}$ in an uncovered glass vial (surface area between dispersion and air was $8 \mathrm{~cm}^{2}$ ). The resulting particles were washed by centrifugation using methanol to remove HD and excess surfactant and then dried under vacuum at room temperature.

\section{Measurements}

The amount of toluene in the dispersion was determined by gas chromatography (Shimadzu Corporation, Kyoto, Japan, GC-2014) with helium as the carrier gas, $\mathrm{N}, \mathrm{N}$-dimethylformamide as a solvent and $p$-xylene as an internal standard. Molecular weight distribution was measured by gel permeation chromatography, with two styrene/divinylbenzene gel columns (TOSOH Corporation, Yamaguchi, Japan, TSK gel $\mathrm{GMH}_{\mathrm{HR}}-\mathrm{H}, 7.8 \mathrm{~mm}$ i.d. $\times 30 \mathrm{~cm}$ ) using tetrahydrofuran as the eluent at $40{ }^{\circ} \mathrm{C}$ at a flow rate of $1.0 \mathrm{ml} \mathrm{min}^{-1}$, employing refractive index (TOSOH Corporation) and ultraviolet detectors (TOSOH Corporation, UV-8II). The columns were calibrated with six standard PS samples $\left(1.05 \times 10^{3}-5.48 \times 10^{6}, M_{\mathrm{w}} / M_{\mathrm{n}}=1.01-1.15\right)$.

\section{Interfacial tension measured by the pendant drop method}

The densities of toluene solutions of PS and HD were measured with a pycnometer (volumetric flask type). Interfacial tensions ( $\gamma_{\text {PS-T/Surf-w }}$ or $\gamma_{\text {HD-T/Surf-w }}$ ) between the PS (or HD)-toluene droplets and various surfactant aqueous solutions as functions of PS (or HD) weight fraction ( $w_{\mathrm{PS}}$ or $w_{\mathrm{HD}}$ ) were measured by the pendant drop method with a Drop Master 500 (Kyowa Interface Science Co., Ltd., Saitama, Japan). All of the measurements were performed at room temperature $\left(\mathrm{ca} .20^{\circ} \mathrm{C}\right)$. The accuracy of the interfacial tensions reported was $\pm 0.1 \mathrm{mN} \mathrm{m}^{-1}$.

\section{Microscope observation}

The dispersions of PS/HD and $\mathrm{P}(\mathrm{S}-\mathrm{PM}) / \mathrm{HD}$ particles were observed with a Nikon (Tokyo, Japan) Eclipse 80i optical microscope and an Olympus (Tokyo, Japan) FV1000-KDM confocal laser scanning microscope, respectively. Dried PS particles were observed with a Hitachi (Tokyo, Japan) S-2460 scanning electron microscope at an acceleration voltage of $15 \mathrm{kV}$.

\section{RESULTS AND DISCUSSION}

The morphology of a PS/HD particle reflects the thermodynamic equilibrium morphology of the phase-separated $\mathrm{PS} / \mathrm{HD} /$ toluene droplet determined by interfacial free energy minimization. Thus, the measurement of each interfacial tension enables prediction of the most stable droplet morphology. However, measurement of $\gamma_{\text {PS-T/Surf-w }}$ at high $w_{\mathrm{PS}}$ by the pendant drop method was difficult because of high viscosity. $\gamma_{\text {PS-T/Surf-w }}$ was thus calculated from the following equations: ${ }^{39}$

$$
\begin{aligned}
\ln \left[\left(\varphi_{P S}^{S} / \varphi_{P S}\right)^{1 / r} /\left(\varphi_{\mathrm{T}}^{S} / \varphi_{\mathrm{T}}\right)\right]= & {\left[\left(\gamma_{\mathrm{T} / \text { Surf-w }}-\gamma_{\mathrm{PS}-\mathrm{T} / \text { Surf-w }}\right) a / k T\right] } \\
+ & \chi(l+m)\left(\varphi_{\mathrm{T}}-\varphi_{\mathrm{PS}}\right)-\chi l\left(\varphi_{\mathrm{T}}^{S}-\varphi_{\mathrm{PS}}^{S}\right) \\
\left(\gamma_{\mathrm{PST} / \text { Surf-w }}-\gamma_{\mathrm{T} / \text { Surf-w }}\right) a / k T= & \ln \left(\varphi_{\mathrm{T}}^{S} / \varphi_{\mathrm{T}}\right)+[(r-1) / r]\left(\varphi_{\mathrm{PS}}^{S}-\varphi_{\mathrm{PS}}\right) \\
& +\chi\left[l\left(\varphi_{\mathrm{PS}}^{S}\right)^{2}-(l+m)\left(\varphi_{\mathrm{PS}}\right)^{2}\right]
\end{aligned}
$$

where, T and Surf-w subscripts denote toluene and surfactant aqueous solutions, respectively, $\varphi_{i}$ and $\varphi_{i}^{\mathrm{S}}$ are the volume fractions of the $i$ th component in the particle and of the $i$ th component at the surface, respectively. $\gamma_{i-T / S u r f-w}$ is the interfacial tension between the $i$ th component and the surfactant aqueous phase, $r$ is the ratio of the component molar volumes $(r=856), a$ is the surface area occupied by a toluene molecule $\left(31.4 \AA^{2}\right), \quad k$ is the Boltzmann constant $\left(1.38 \times 10^{-23} \mathrm{JK}^{-1}\right), \quad T$ is the temperature $(300 \mathrm{~K}), \quad \chi$ is the interaction parameter between PS and toluene (0.40) and $l$ and $m$ are constants $(l=0.5$ and $m=0.25) \cdot{ }^{40-42}$ Equations 1 and 2 give the interfacial tension between the PS and surfactant aqueous phases $\left(\gamma_{\text {PS-T/Surf-w }}\right)$ as a function of $\varphi_{\text {PS. The }} \chi$ parameter between PS and toluene depends on the polymer concentration. These effects were not considered in the calculation of interfacial tensions, and as assumed previously, ${ }^{38}$ the $\chi$ parameter was treated as constant in the current study.

Figure 1 shows $\gamma_{\text {PS-T/Surf-w }}$ and $\gamma_{\text {HD-T/Surf-w }}$ with various types of surfactants as functions of $w_{\mathrm{PS}}$ and $w_{\mathrm{HD}}$. The $\gamma_{\mathrm{PS}-\mathrm{T} / \mathrm{Surf}-\mathrm{w}}$ values calculated based on the above equations indicate constant values at low $w_{\mathrm{PS}}$ and were consistent in all cases with those measured by the pendant drop method at $w_{\mathrm{PS}}=0.17$. At high $w_{\mathrm{PS}}, \gamma_{\mathrm{PS}-\mathrm{T} / \mathrm{Surf}-\mathrm{w}}$ drastically increased with increasing $w_{\mathrm{PS}}$. On the other hand, $\gamma_{\text {HD-T/Surf-w }}$ gradually increased with increasing $w_{\mathrm{HD}}$. The magnitude relations of $\gamma_{\text {PS-T/Surf-w }}$ and $\gamma_{\text {HD-T/Surf-w }}$ were reversed in the cases of PVA, Emulgen 931 and Emulgen 950.

Morphological development of phase-separated PS/HD/toluene droplets associated with intergradations of the interfacial tensions is retarded by increasing viscosity inside the droplet because of increasing polymer fraction (i.e., evaporation of toluene) because the glass transition temperature $\left(T_{\mathrm{g}}\right)$ of PS is well above the room temperature. The $T_{\mathrm{g}}$ of the PS-toluene phase in the phase-separated droplet was calculated with the Fox equation, ${ }^{43}$ assuming that the melting temperature of toluene is its $T_{\mathrm{g}}\left(-93^{\circ} \mathrm{C}\right)$. Because the $T_{\mathrm{g}}$ of the PS-toluene phase reached room temperature at $w_{\mathrm{PS}}=0.75$, the $w_{\text {PS }}$ where the droplet morphology is fixed was defined as 0.75 in this experiment.

Table 1 shows $\gamma_{\text {PS-T/Surf-w }}$ and $\gamma_{\text {HD-T/Surf-w }}$ with various types of surfactants at $w_{\mathrm{PS}}$ and $w_{\mathrm{HD}}=0.75$; the difference between $\gamma_{\mathrm{PS}-\mathrm{T} / \mathrm{Surf}-\mathrm{w}}$ and $\gamma_{\text {HD-T/Surf-w }}(\Delta \gamma)$ decreased in the order Emulgen $930>$ SDS $>$ Emulgen $931>$ Emulgen $950>$ PVA. The thermodynamically stable morphology of phase-separated $\mathrm{PS} / \mathrm{HD} /$ toluene droplets is expected to change from PS-core/HD-shell to $\mathrm{HD}$-core/PS-shell when $\Delta \gamma$ decreases from positive to negative. The resulting particle morphology, which reflects the thermodynamically stable droplet morphology, should also exhibit the same tendency.
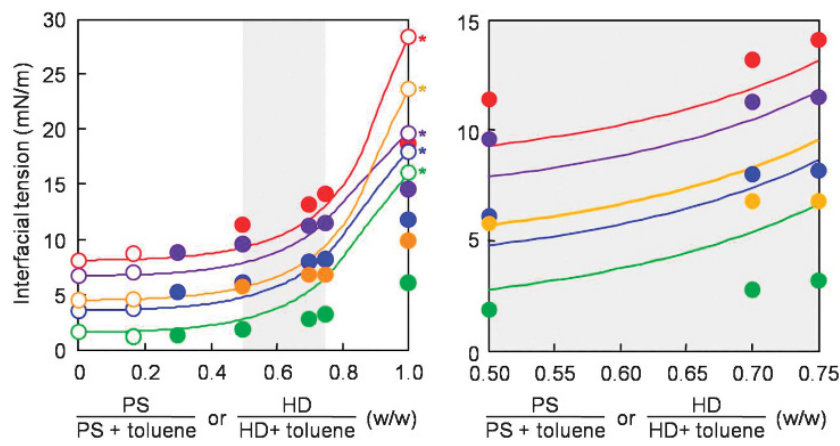

Figure 1 Interfacial tensions between $0.33 \mathrm{wt} \%$ surfactant aqueous solutions and toluene droplets containing dissolved PS $(\bigcirc, \bigcirc, \bigcirc, \bigcirc, \bigcirc)$ or HD $(9,0)$ measured by the pendant drop method as functions of PS or HD weight fraction. Surfactants: $(O, 0)$ PVA; $(\bigcirc, 0)$ SDS; $(\bigcirc, \bigcirc)$ Emulgen 930; $(\bigcirc, \bigcirc)$ Emulgen 931; $(\bigcirc, \bigcirc)$ Emulgen 950. Asterisks denote calculated values using the Young equation based on contact angles of surfactant aqueous solutions on a PS film. The lines are the interfacial tensions between $0.33 \mathrm{wt} \%$ surfactant aqueous solutions (corresponding to each color) and PS-containing toluene droplets calculated using Equations 1 and 2. 
Table 1 Interfacial tension between 0.33 wt\% surfactant aqueous solutions and toluene (T) droplets dissolving of PS or HD (PS or HD)/ toluene $=3 / 1, w / w)$

\begin{tabular}{lrrrrr}
\hline & \multicolumn{5}{c}{ Interfacial tension $\left(\mathrm{mN} \mathrm{m}^{-1}\right)$} \\
\cline { 2 - 6 } & SDS & Emulgen 930 & Emulgen 931 & Emulgen 950 & PVA \\
\hline$\gamma_{\text {PS-T/Surf-w }}{ }^{\mathrm{a}}$ & 9.5 & $<6.6$ & 8.6 & 11.7 & 13.1 \\
$\gamma_{\text {HD-T/Surf-w }}{ }^{\mathrm{b}}$ & 6.8 & 3.2 & 8.2 & 11.5 & 14.1 \\
$\Delta \gamma^{\mathrm{C}}$ & 2.7 & $<3.4$ & 0.4 & 0.2 & -1.0 \\
\hline
\end{tabular}

Abbreviations: HD, hexadecane; PS, polystyrene.

aCalculated interfacial tension according to Siow et al..$^{39}$

bMeasured by the pendant drop method.

${ }^{c} \Delta \gamma=\gamma_{\text {PS-T/Surf-w }}-\gamma_{\text {HD-T/Surf-w }}$
Figure 2 shows optical micrographs of PS/HD particles prepared by the solvent evaporation method. The morphologies of the resulting particles depended on the type of surfactant. To distinguish between the PS and HD phases, $\mathrm{P}(\mathrm{S}-\mathrm{PM})$ incorporating a fluorescent unit was employed instead of PS. It was confirmed that a very small amount of copolymerized fluorescent PM did not affect the particle morphology ${ }^{44}$ (i.e., PS/HD and $\mathrm{P}(\mathrm{S}-\mathrm{PM}) / \mathrm{HD}$ particles had the same morphology).

Figure 3 shows confocal laser scanning micrographs of $\mathrm{P}(\mathrm{S}-\mathrm{PM}) /$ HD particles prepared under the same conditions as those in Figure 2. The interfacial area between the $\mathrm{P}(\mathrm{S}-\mathrm{PM})$ phase and the aqueous media increased with decreasing $\Delta \gamma$, except in the cases of SDS and Emulgen 930 (see Table 1).

Previous work ${ }^{37}$ has shown that a non-ionic surfactant (polyoxyethylene nonylphenylether with an average ethylene oxide
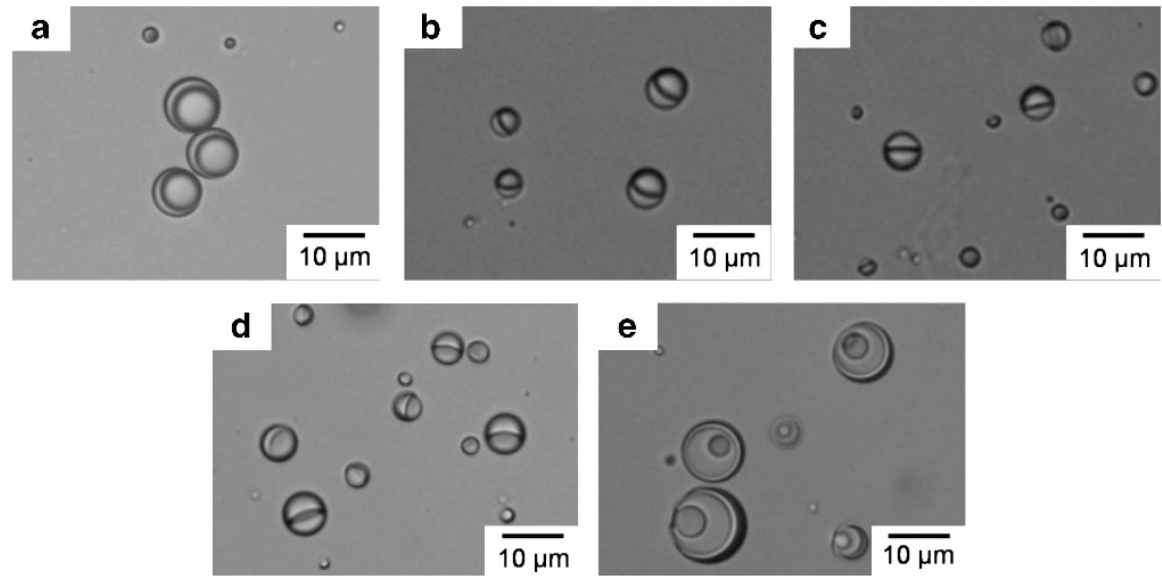

Figure 2 Optical micrographs of PS/HD particles prepared by slow release of toluene from PS/HD/toluene (1/1/29, v/v/v) droplets dispersed in 0.33 wt\% aqueous solutions of SDS (a), Emulgen 930 (b), Emulgen 931 (c), Emulgen 950 (d) and PVA (e).
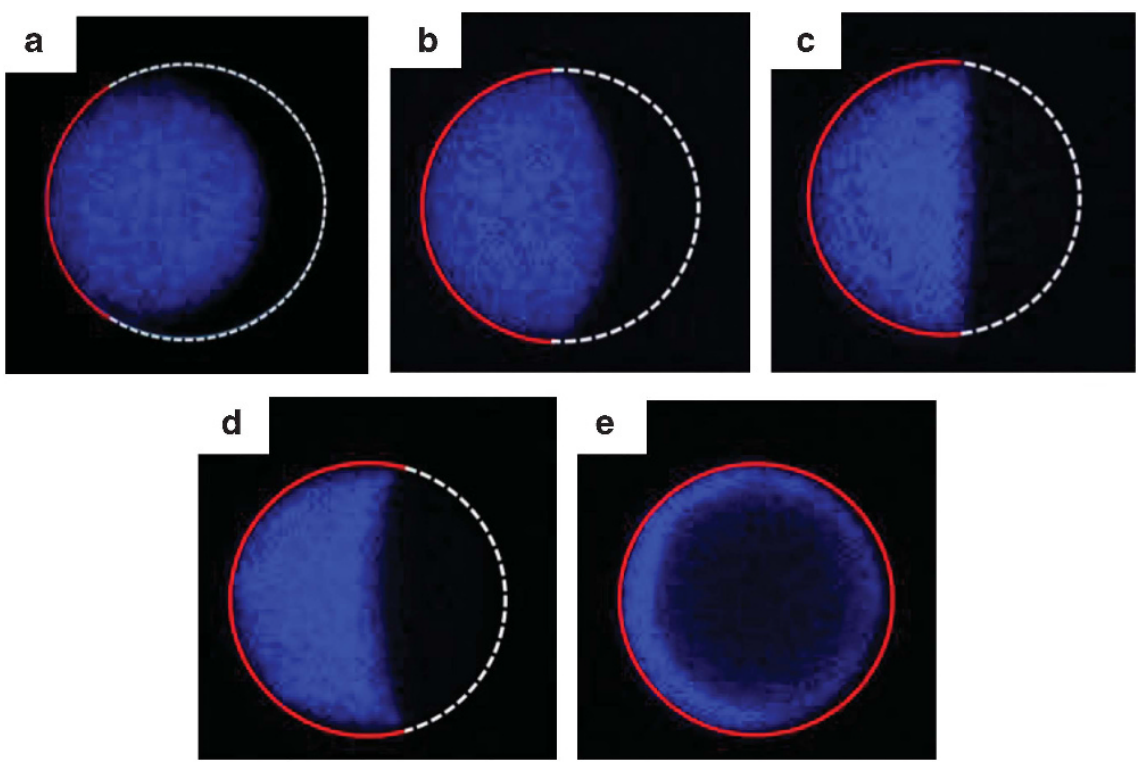

Figure 3 Confocal laser scanning micrographs of P(S-PM)/HD particles prepared by slow release of toluene from P(S-PM)/HD/toluene (1/1/29, v/v/v) droplets dispersed in 0.33 wt\% aqueous solutions of SDS (a), Emulgen 930 (b), Emulgen 931 (c), Emulgen 950 (d) and PVA (e). The red and white curve lines indicate interfaces of the P(S-PM) and HD phases, respectively, contacting with each surfactant aqueous medium. 
chain length of 10.9) dissolved into both the oil and aqueous phases and significantly reduced the oil-aqueous medium interfacial tension.

Figure 4 shows the variation of Emulgen concentrations in aqueous media as a function of the toluene weight fraction relative to the total amount of toluene, water and Emulgen. Emulgen 930, with an average ethylene oxide unit length of 15.4, dissolved into the toluene phase whereas nearly all Emulgen 931 and 950 dissolved in the aqueous phases. The effect of partitioning was not considered in the equations calculating $\gamma_{\text {PS-T/Surf-w }}$; the actual $\gamma_{\text {PS-T/Surf-w }}$ would thus be

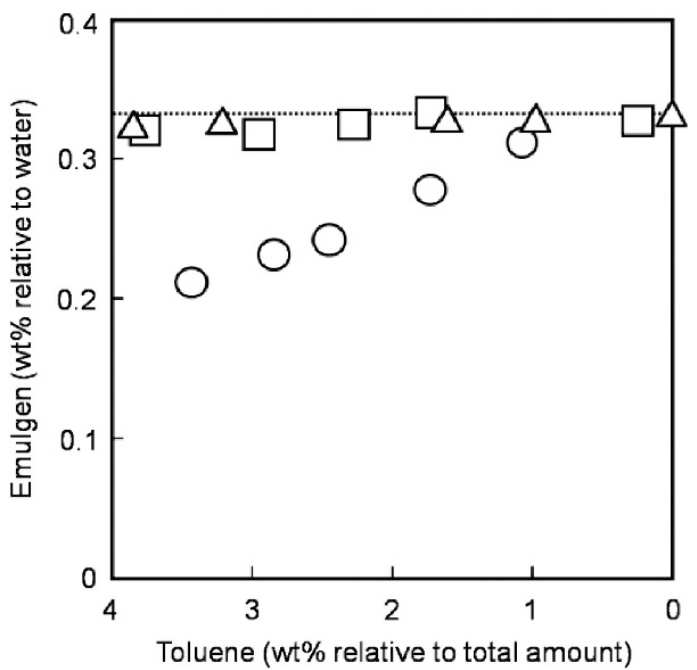

Figure 4 Emulgen loading content in the aqueous phases as a function of toluene content (relative to total amount of toluene, water and Emulgen) during evaporation of toluene from dispersions of toluene and $0.33 \mathrm{wt} \%$ Emulgen aqueous solution (toluene/water $=4 / 100, w / w)$. Emulgen: $(O)$ 930; $(\square)$ 931; $(\triangle)$ 950. Dotted line shows the initial Emulgen concentration. lower than the calculated one in the Emulgen 930 case, resulting in a lower $\Delta \gamma$ for Emulgen 930 than for SDS. This could explain the unexpected results (a) and (b) in Figure 3.

Figure 5 shows scanning electron microscope photographs of PS particles after rapid removal of HD with methanol from the PS/HD particles shown in Figure 2. PS particles with various shapes corresponding to the phase-separated morphology were formed. PS particles prepared using PVA had a single deep dimple at the surface; this was attributed to the volume reduction of the HD phase after hardening of the PS phase during toluene evaporation from the droplets. ${ }^{36}$ In particular, hemispherical PS particles were obtained when Emulgen 931 was employed. Such particles can be oriented on planar surfaces, with their curved surfaces pointing upward and can act as microlens. ${ }^{45,46}$ This simple preparation method should be applicable to other polymer and poor solvent systems, and can thus be a very useful tool for the preparation of hemispherical particles for industrial applications.

\section{CONCLUSIONS}

Various nonspherical PS particles were prepared by a rapid removal of HD from phase-separated PS/HD particles obtained by the slow release of toluene from $\mathrm{PS} / \mathrm{HD} /$ toluene droplets dispersed in various surfactant aqueous solutions at room temperature. The phaseseparated morphology of the PS/HD particles and the final PS particle shape could be controlled by tuning $\gamma_{\text {PS-T/Surf-w }}$ and $\gamma_{\text {HD-T/ }}$ Surf-w. As a result, hemispherical PS particles were obtained from the phase-separated PS/HD/toluene droplets having a Janus structure when Emulgen 931 was used as the surfactant.

\section{ACKNOWLEDGEMENTS}

This work was supported by a Grant-in-Aid for Scientific Research (Grant 21245050) from the Japan Society for the Promotion of Science (JSPS). Part CCCL of the series 'Studies on Suspension and Emulsion'
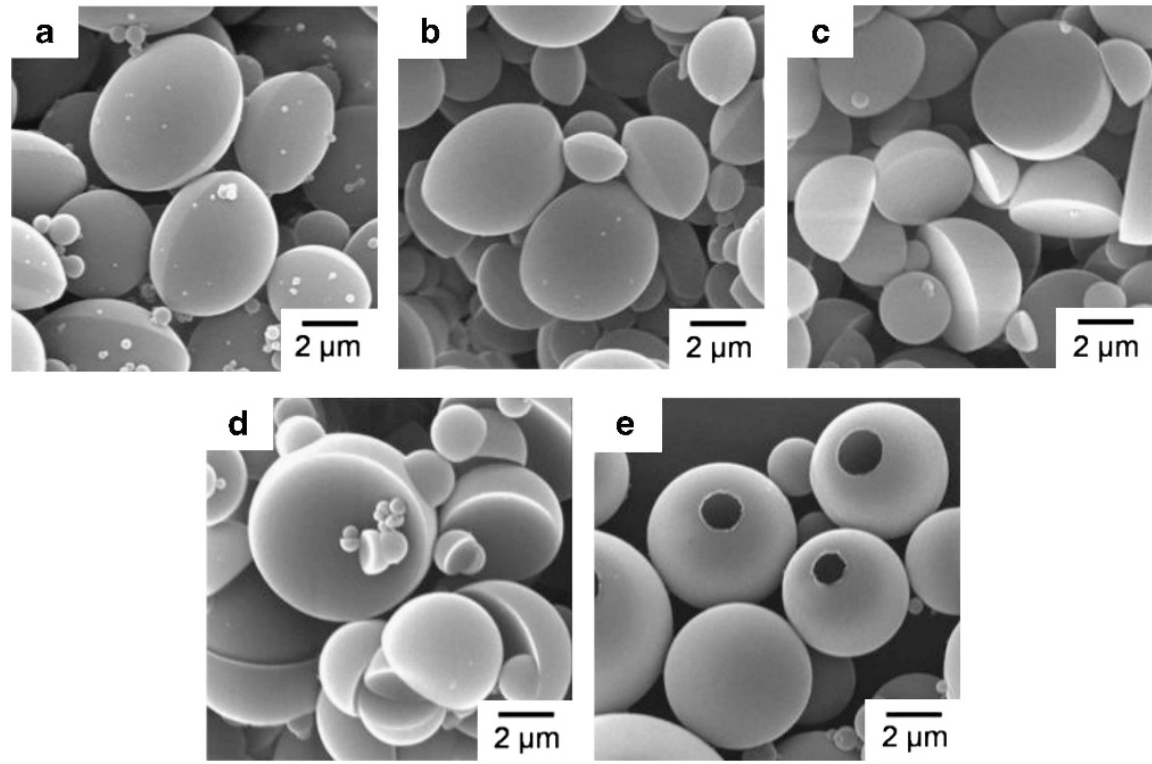

Figure 5 Scanning electron microscope micrographs of PS particles after the rapid removal of HD with methanol from PS/HD particles prepared by the slow release of toluene from PS/HD/toluene (1/1/29, v/v/v) droplets dispersed in 0.33 wt \% aqueous solutions of SDS (a), Emulgen 930 (b), Emulgen 931 (c), Emulgen 950 (d) and PVA (e). 
1 Lu, Y., Yin, Y., Li, Z.-Y. \& Xia, Y. Colloidal crystals made of polystyrene spheroids: fabrication and structural/optical characterization. Langmuir 18, 7722 7727 (2002).

2 Mock, E. B. \& Zukoski, C. F. Determination of static microstructure of dilute and concentrated suspension of anisotropic particles by ultra-small-angle X-ray scattering. Langmuir 23, 8760-8771 (2007).

3 Hosein, I. D. \& Liddell, C. M. Convectively assembled nonspherical mushroom capbased colloidal crystals. Langmuir 23, 8810-8814 (2007).

4 Mishchenko, M. I., Hovenier, J. W. \& Travis, L. D. Light scattering by nonspherical particles: theory, measurements, and applications (Academic Press, San Diego, CA, 2000)

5 Jogun, S. M. \& Zukoski, C. F. Reology and microstructure of dense suspensions of plate-shaped colloidal particles. J. Rheol. 43, 847-871 (1999).

6 Ho, C. C., Ottewill, R. H. \& Yu, L. Examination of ellipsoidal polystyrene particles by electrophoresis. Langmuir 13, 1925-1930 (1997)

7 Matsumoto, T., Okubo, M. \& Shibao, S. Formation mechanism of heterogeneous structure in particles produced by seeded emulsion polymerization. Kobunshi Ronbunshu 33, 575-583 (1976).

8 Okubo, M., Ando, M., Yamada, A., Katsuta, Y. \& Matsumoto, T. Anomalous composite polymer emulsion particles with voids produced by seeded emulsion polymerization. $J$. Polym. Sci. Polym. Lett. Ed. 19, 143-147 (1981).

9 Okubo, M., Katsuta, Y. \& Matsumoto, T. Rupture of anomalous composite particles prepared by seeded emulsion polymerization in aging period. J. Polym. Sci. Polym. Lett. Ed. 20, 45-51 (1982).

10 Cho, I. \& Lee, K.-W. Morphology of latex particles formed by poly(methyl methacrylate)seeded emulsion polymerization of styrene. J. Appl. Polym. Sci. 30, 1903-1926 (1985).

11 Skjeltorp, A. T., Ugelstad, J. \& Ellingsen, T. Preparation of nonspherical, monodisperse polymer particles and their self-organization. J. Colloid Interface. Sci. 113, 577-582 (1986).

12 Okubo, M., Kanaida, K. \& Matsumoto, T. Production of anomalously shaped carboxylated polymer particles by seeded emulsion polymerization. Colloid Polym. Sci. 265, 876-881 (1987).

13 Okubo, M. Control of particle morphology in emulsion polymerization. Makromol. Chem., Macromol. Symp. 35/36, 307-325 (1990).

14 Sheu, H. R., El-Aasser, M. S. \& Vanderhoff, J. W. Phase separation in polystyrene latex interpenetrating polymer networks. J. Polym. Sci. Part A: Polym. Chem. 28, 629-651 (1990).

15 Okubo, M., Fujiwara, T. \& Yamaguchi, A. Morphology of anomalous polystyrene/ polybutyl acrylate composite particles produced by seeded emulsion polymerization. Colloid. Polym. Sci. 276, 186-189 (1998).

$16 \mathrm{Ni}$, H., Ma, G., Nagai, M. \& Omi, S. Novel method of preparation of a charged mosaic membrane by using dipole-like microspheres. II. Preparation of dumbbell/egg-like microspheres. J. Appl. Polym. Sci. 80, 2002-2017 (2001).

17 Okubo, M., Wang, Z., Yamashita, T., Ise, E. \& Minami, H. Morphology of micron-sized, monomer-adsorbed, cross-linked polymer particles having snowman shape prepared by the dynamic swelling method. J. Polym. Sci. Part A: Polym. Chem. 39, 3106-311 (2001).

18 Okubo, M., Takekoh, R. \& Suzuki, A. Preparation of micron-sized, monodisperse poly(methyl methacrylate)/polystyrene composite particles having a large number of dents on their surfaces by seeded dispersion polymerization in the presence of decalin. Colloid. Polym. Sci. 280, 1057-1061 (2002).

19 Wang, D., Dimonie, V. L., Sudol, E. D. \& El-Aasser, M. S. Seeded dispersion polymerization. J. Appl. Polym. Sci. 84, 2710-2720 (2002).

20 Kegel, W. K., Breed, D., Elsesser, M. \& Pine, D. J. Formation of anisotropic polymer colloids by disparate relaxation times. Langmuir 22, 7135-7136 (2006).

21 Kim, J.-W., Larsen, R. J. \& Weitz, D. A. Synthesis of nonspherical colloidal particles with anisotropic properties. J. Am. Chem. Soc. 128, 14374-14377 (2006)

22 Mock, E. B., De Bruyn, H., Howkett, B. S., Gilbert, R. G. \& Zukoski, C. F. Synthesis of anisotropic nanoparticles by seeded emulsion polymerization. Langmuir 22 4037-4043 (2006).
23 Fujibayashi, T. \& Okubo, M. Preparation and thermodynamic stability of micron-sized monodisperse composite polymer particles of disc-like shapes by seeded dispersion polymerization. Langmuir 23, 7958-7962 (2007).

24 Yang, S.-M., Kim, S.-H. \& Yi, G.-R. Synthesis of assembly of structured colloidal particles. J. Mater. Chem. 18, 2177-2190 (2008)

25 Dendukuri, D., Tsoi, K., Hatton, T. A. \& Doyle, P. S. Controlled synthesis of nonspherical microparticles using microfluidics. Langmuir 21, 2113-2116 (2005).

26 Nie, Z., Xu, S., Seo, M., Lewis, P. C. \& Kumacheva, E. Polymer particles with various shapes and morphologies produced in continuous microfluidic reactors. J. Am. Chem Soc. 127, 8058-8063 (2005).

27 Xu, S., Nie, Z., Seo, M., Lewis, P. C., Kumacheva, E., Stone, H. A., Garstecki, P., Weibel, D. B., Gitlin, I. \& Whitesides, G. M. Generation of monodisperse particles by using microfluidics: control over size, shape, and composition. Angew. Chem. Int. Ed. 44, 724-728 (2005)

28 Nisisako, T., Torii, T., Takahashi, T. \& Takizawa, Y. Synthesis of monodisperse bicolored janus particles with electrical anisotropy using a microfluidic co-flow system. Adv. Mater. 18, 1152-1156 (2006).

29 Ho, C. C., Keller, A., Odell, J. A. \& Ottewill, R. H. Preparation of monodisperse ellipsoidal polystyrene particles. Colloid. Polym. Sci. 271, 469-479 (1993).

30 Alargova, R. G., Bhatt, K. H., Paunov, V. N. \& Velev, O. D. Scalable synthesis of a new class of polymer microrods by a liquid-liquid dispersion technique. Adv. Mater. 16, 1653-1657 (2004)

31 Sun, Z. Q., Chen, X., Zhang, J. H., Chen, Z. M., Zhang, K., Yan, X., Wang, Y. F., Yu, W. Z. \& Yang, B. Langmuir 21, 8987-8991 (2005)

32 Okubo, M., Ichikawa, K., Tsujihiro, M. \& He, Y. Production of anomalous polyme microspheres having uneven surfaces by 'stepwise' heterocoagulation technique, Colloid. Polym. Sci. 268, 791-796 (1990).

33 Okubo, M., He, Y. \& Ichikawa, K. Analysis of "stepwise" heterocoagulation process of small cationic polymer particles onto large anionic polymer particles using dynamic light scattering. Colloid. Polym. Sci. 269, 125-130 (1991).

34 Tajima, A., Higuchi, T., Yabu, H. \& Shimomura, M. Hemispherical polymer nanoparticles of polyisoprene-poly(methyl methacrylate) blend with core-shell structure. Colloids Surf. A. 313-314, 332-334 (2008).

35 Tanaka, T., Komatsu, Y., Fujibayashi, T., Minami, H. \& Okubo, M. A novel approach for preparation of micrometer-sized, monodisperse dimple and hemispherical polystyrene particles. Langmuir 26, 3848-3853 (2010).

36 Saito, N., Kagari, Y. \& Okubo, M. Effect of colloidal stabilizer on the shape of PS/PMMA composite particles prepared by in aqueous medium by the SR method. Langmuir 22, 9397-9402 (2006).

37 Saito, N., Nakatsuru, R., Kagari, Y. \& Okubo, M. Formation of 'Snowman-like' PS/PMMA/Toluene droplets dispersed in an aqueous solution of a nonionic surfactant at thermodynamic equilibrium. Langmuir 23, 11506-11512 (2007).

38 Tanaka, T., Nakatsuru, R., Kagari, Y., Saito, N. \& Okubo, M. Effect of molecular weight on the morphology of polystyrene/poly(methyl methacrylate) composite particles prepared by the solvent evaporation method. Langmuir 24, 12267-12271 (2008).

39 Siow, K. S. \& Patterson, D. Surface thermodynamics of polymer-solutions. J. Phys. Chem. 77, 356-365 (1973)

40 Prigogine, I. \& Marechal, J. The influence of differences in molecular size on the surface tension of solutions. IV. J. Colloid Sci 7, 122-127 (1952).

41 Defay, R., Prigogine, I., Bellemans, A. \& Everett, D. H. Surface Tension of Solutions Mixtures of Different Sizes; Surface Tension and Adsorption 199-207 (Wiley, London, 1966)

42 Schuld, N. \& Wolf, B. A. Polymer-Solvent Interaction Parameters Number of VII/247264 (John Wiley \& Sons, 1999).

43 Fox, T. G. Bull. Am. Phys. Soc. 1, 123 (1956).

44 Saito, N., Kagari, Y. \& Okubo, M. Revisiting the morphology development of solventswollen composite polymer particles at thermodynamic equilibrium. Langmuir 23 5914-5919 (2007).

$45 \mathrm{Lu}$, Y., Yin, Y \& Xia, Y. A self-assembly approach to the fabrication of patterned, twodimensional arrays of microlenses of organic polymers. Adv. Mater 13, 34-37 (2001).

46 Sakurai, Y., Okuda, S., Nagayama, N. \& Yokoyama, M. Novel microlens array fabrication utilizing UV-photodecomposition of polysilane. J. Mater. Chem. 11, 1077-1080 (2001) 